\title{
DNA-encoded bispecific T cell engagers and antibodies present long-term antitumor activity
}

\author{
Alfredo Perales-Puchalt, ${ }^{1}$ Elizabeth K. Duperret, ${ }^{1}$ Xue Yang, ${ }^{,}$Patricia Hernandez, ${ }^{1}$ \\ Krzysztof Wojtak, ${ }^{1}$ Xizhou Zhu, ${ }^{1}$ Seang-Hwan Jung, ${ }^{1}$ Edgar Tello-Ruiz, ${ }^{1}$ Megan C. Wise, ${ }^{2}$ \\ Luis J. Montaner, ${ }^{1}$ Kar Muthumani, ${ }^{1}$ and David B. Weiner ${ }^{1}$ \\ 'Vaccine and Immunotherapy Center, The Wistar Institute, Philadelphia, Pennsylvania, USA. 'Inovio Pharmaceuticals, \\ Plymouth Meeting, Pennsylvania, USA.
}

\begin{abstract}
Specific antibody therapy, including mAbs and bispecific T cell engagers (BiTEs), are important new tools for cancer immunotherapy. However, these approaches are slow to develop and may be limited in their production, thus restricting the patients who can access these treatments. BiTEs exhibit a particularly short half-life and difficult production. The development of an approach allowing simplified development, delivery, and in vivo production would be an important advance. Here we describe the development of a designed synthetic DNA plasmid, which we optimized to permit high expression of an anti-HER2 antibody (HER2dMAb) and delivered it into animals through adaptive electroporation. HER2dMAb was efficiently expressed in vitro and in vivo, reaching levels of $50 \mu \mathrm{g} / \mathrm{ml}$ in mouse sera. Mechanistically, HER2dMAb blocked HER2 signaling and induced antibody-dependent cytotoxicity. HER2dMAb delayed tumor progression for HER2expressing ovarian and breast cancer models. We next used the HER2dMAb single-chain variable fragment portion to engineer a DNA-encoded BiTE (DBiTE). This HER2DBiTE was expressed in vivo for approximately 4 months after a single administration. The HER2DBiTE was highly cytolytic and delayed cancer progression in mice. These studies illustrate an approach to generate DBiTEs in vivo, which represent promising immunotherapies for HER2+ tumors, including ovarian and potentially other cancers.
\end{abstract}

Conflict of interest: DBW has received a sponsored research agreement (SRA) from, has an ownership interest in, has patents filed with, and has received consulting and received speakers honoraria from Inovio; has received an SRA and consulting and speakers honoraria from GeneOne and Geneos; and has served on advisory boards for AstraZeneca and Sanofi. MCW is an employee at Inovio Pharmaceuticals.

Copyright: () 2019 American Society for Clinical Investigation

Submitted: November 8, 2018

Accepted: March 7, 2019

Published: April 18, 2019.

Reference information: JCI Insight. 2019;4(8):e126086. https://doi. org/10.1172/jici.insight.126086

\section{Introduction}

Monoclonal antibody therapy has been a game changer for cancer therapy, including treatments for lymphoma or human epidermal growth factor receptor $2^{+}\left(\right.$HER2 $\left.{ }^{+}\right)$breast cancer $(1,2)$. However, this approach has limitations, including slow development timelines, limited modification options, and short half-life for the molecules, requiring frequent repeat administrations. These limitations contribute to the high cost of development and deployment of these important tools and represents a challenge for the development of combination approaches. An important recent advance in the field of monoclonal technology is the bispecific T cell engager (BiTE), which combines the specificity of mAbs with the cytotoxic potential of $\mathrm{T}$ cells. BiTEs have shown promising results in leukemia clinical trials (3); however, this therapy has a limited applicability because it requires continuous intravenous infusion for weeks (4) and has manufacturing limitations. A longer-lived, simpler, reproducible production method for these antibody-based products would be important for cancer immunotherapy.

Plasmid DNA-encoded antibodies (dMAbs) have been recently explored to treat infectious diseases (5-7) and cancer (7-9). This approach presents unique advantages over conventional protein antibodies and those encoded in viral vectors for in vivo delivery, including simplicity of engineering and delivery, short development times and production, and longer in vivo expression without gene integration. Previous attempts to deliver DNA-encoded HER2 antibodies have resulted in functionally low levels of expression (10); however, novel design, delivery, and formulation improvements should permit higher and longer expression.

In this manuscript, we describe the development of a high-expressing dMAb targeting HER2 and then the modification of this new molecule to develop a potentially novel BiTE targeting HER2. We study these molecules in therapeutic models for the treatment of ovarian and breast cancer. We show that 
both constructs express at high levels in vitro and in vivo for approximately 4 months. HER2dMAb binds to HER2 and induces HER2 signaling blockade and antibody-dependent cellular cytotoxicity. HER2 DNA-encoded BiTE (HER2DBiTE) effectively induces $\mathrm{T}$ cell cytotoxicity against HER2+ tumor cells, resulting in a high proportion of tumor regressions. These potentially novel synthetic DNA approaches represent new tools for further study of immunotherapy for cancer.

\section{Results}

Design and expression of HER2dMAb. To develop a HER2dMAb, we encoded codon and RNA-optimized sequences for the heavy and light chains of pertuzumab into a modified pVAX plasmid expression vector (Figure 1A). We tested antibody expression in vitro by transfecting 293T cells with the synthetic HER2dMAb DNA or an irrelevant DNA-encoded protein. We collected the supernatant and performed Western blot analysis 48 hours later. We easily identified bands corresponding to the heavy and light antibody chains in the HER2dMAb-transfected 293T supernatant but not in the irrelevant protein control (Figure 1B). HER2dMAb was expressed at 5-6 $\mu \mathrm{g} / \mathrm{ml}$, which we validated using RD cells (Supplemental Figure 1A; supplemental material available online with this article; https://doi.org/10.1172/jci.insight.126086DS1).

After confirming in vitro expression, we studied HER2dMAb's in vivo expression. We injected $200 \mu \mathrm{g}$ of HER2dMAb or empty vector followed by adaptive electroporation into the tibialis anterior muscle of mice. We identified the presence of human IgG in sera from the HER2dMAb-injected mice but not in the controls (Figure 1C), with levels of expression as high as $50 \mu \mathrm{g} / \mathrm{ml}$ in mouse sera (Figure 1D). Expression lasted for more than 9 months (Figure 1D).

Next, we examined if this DNA-encoded human IgG would bind HER2. We coated plates with HER2 protein and incubated it with sera from the HER2dMAb-treated mice or control sera. HER2dMAbs from mouse sera bound to HER2 in a dose-dependent manner (Figure 1E). To confirm HER2 binding when the protein is present on the cell surface, we overexpressed HER2 in the murine cell line Brpkp110. HER2dMAb bound HER2 by flow cytometry only when ectopically expressed (Figure 1F).

HER2 is expressed in human ovarian cancer cell lines. HER2 is overexpressed (histological score $2+$ or $3+$ ) in approximately $11.4 \%$ of ovarian cancers (11). To determine whether HER2 is also expressed in ovarian cancer cell lines, we performed flow cytometry using a commercial 24D2 antibody (Figure 2A). We then validated the binding of our HER2dMAb by doing flow cytometry to these same cells (Figure 2B). To further validate the in vivo expression and potential targeting of ovarian cancer cell lines using our HER2dMAb, we generated OVCAR3 tumors in mice and performed immunofluorescence on frozen tumor sections. We found positive binding using sera from HER2dMAb-transfected mice but not with control sera, confirming HER2's in vivo expression and relevant binding of HER2dMAb (Figure 2C).

HER2AMAb mediates HER2 signaling blockade and antibody-dependent cellular cytotoxicity. Different mechanisms have been attributed to the antitumor effects of anticancer antibodies. Pertuzumab acts by preventing HER2 heterodimerization and agonist-mediated signaling (12). As expected, HER2dMAb prevented HER2-HER3 agonist heregulin-induced (HRG-induced) signaling in OVCAR3 cells, as evidenced by decreased Akt phosphorylation when compared with the vehicle control (Figure 3A), supporting its conserved mechanism of action. The reduction in p-Akt was stronger in the presence of HRG than in its absence, supporting the action through HER2-HER3 dimerization inhibition, but never completely, because HER2 activation is only one of multiple mechanisms inducing the activation of the Akt pathway.

Another mechanism by which mAbs have antitumor activity is through antibody-dependent cellular cytotoxicity (ADCC). To study the ADCC potential of HER2dMAb, we incubated OVCAR3 cells with or without peripheral blood mononuclear cells (PBMCs), in the presence of sera from HER2dMAb- or empty vector-treated mice. HER2dMAb sera effectively killed the ovarian cancer cells in the presence of PBMCs, similar to commercially available Hu4D5, but not in their absence. No killing was observed in the control sera conditions (Figure 3B and Supplemental Figure 1B) or against HER2 ${ }^{-}$cell lines, such as MDA-MB-231 (Supplemental Figure 1C). Similarly, HER2dMAb showed antibody-dependent phagocytosis activity (Supplemental Figure 1D).

HER $2 \mathrm{dMAb}$ delays cancer progression in vivo. To determine the antitumor effects of HER $2 \mathrm{dMAb}$ in vivo, we challenged nude mice with the OVCAR3 ovarian cancer cell line. Nude mice have no T cells but present enhanced NK and macrophage activity (13), and their splenocytes can lyse OVCAR3 in vitro in the presence of HER2dMAb (Supplemental Figure 1E). We delivered $100 \mu \mathrm{g}$ of HER2dMAb or empty vector in 


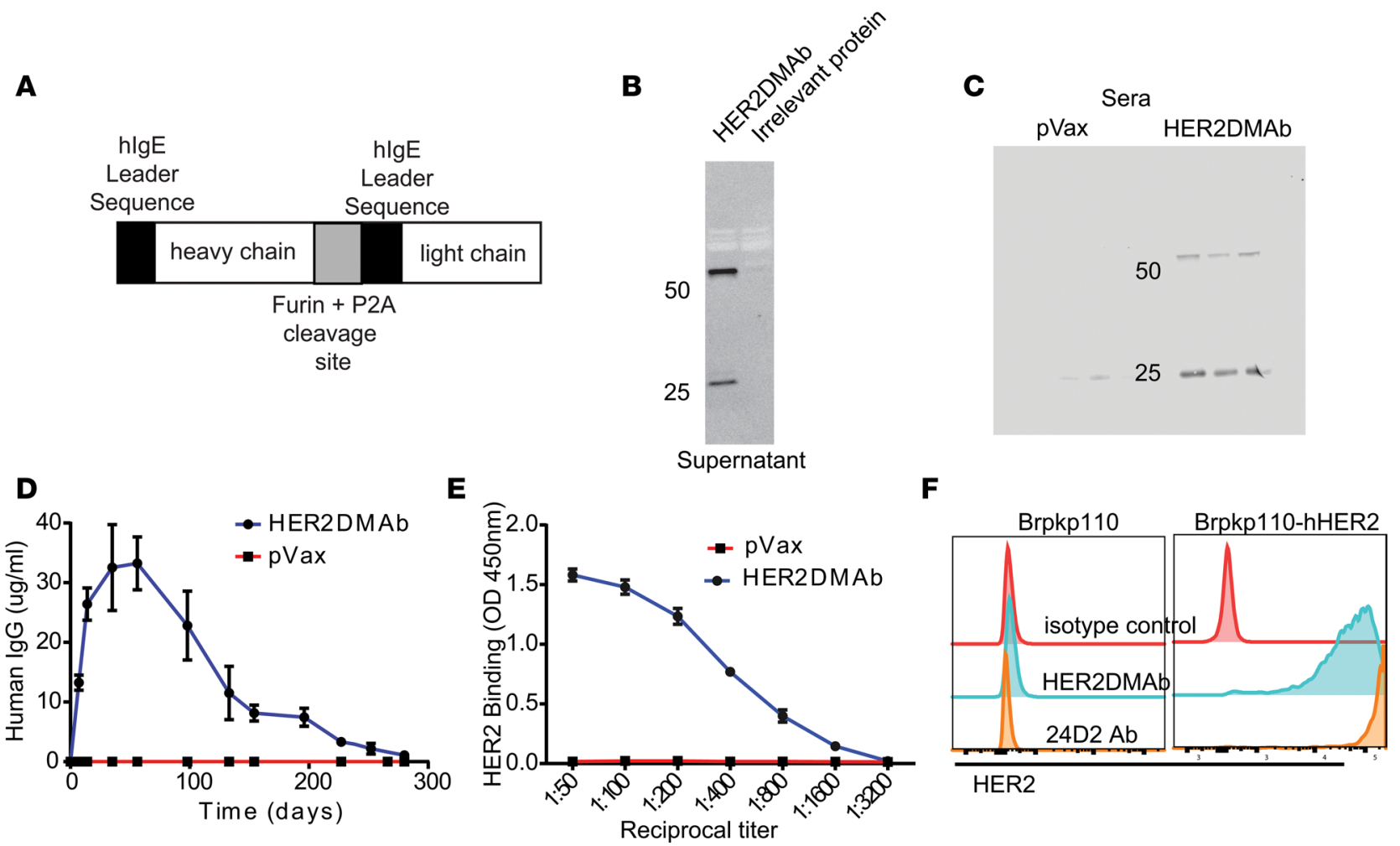

Figure 1. Design, expression, and binding of HER2dMAb. (A) Schematic of DNA construct encoding HER2dMAb. (B) Western blot analysis of HER2dMAb or FSHR constructs expressed in 293T cells ( 2 independent experiments). Numbers indicate molecular weight (kDa). (C) Western blot of human IgG from mouse sera electroporated with HER2dMAb or pVAX control 14 days after DNA injection and electroporation ( $n=5$ mice per group). (D) Expression levels of human IgG quantified by ELISA from sera of nude mice electroporated with HER2dMAb ( $n=5$ mice per group, 2 independent experiments). (E) Binding ELISA of sera from mice expressing HER2dMAb or pVax after coating the plate with HER2 protein ( $n=5$ mice per group, 2 independent experiments). (F) Flow cytometry plot showing binding of HER2dMAb to mouse breast cancer cell lines with and without HER2 expression (representative of triplicates).

the muscle by electroporation when tumors reached an average of $50 \mathrm{~mm}^{3}$. HER2dMAb-injected animals demonstrated a significant delay in tumor growth, resulting in improved survival (Figure 3C). HER2dMAb levels peaked 2 weeks after dMAb injection, with levels of around $20 \mu \mathrm{g} / \mathrm{ml}$, and sustained levels of around $5-10 \mu \mathrm{g} / \mathrm{ml}$ over a month through the end of the experiment (Figure 3D). To validate the antitumor effect in an immunocompetent host, we generated tumors using the murine HER2 ${ }^{+}$breast cancer cell line Brkpk110 (with the caveat of the spontaneous immunogenicity of HER2 in mice). We engineered this cell line to express similar HER2 levels to OVCAR3 (Figure 3E). We treated the mice with HER2dMAb or the empty vector 5 days after tumor challenge. HER $2 \mathrm{dMAb}$ also delayed tumor progression in this aggressive model of breast cancer (Figure 3F).

Upon studying the kinetics of HER2dMAb, we noted the decrease of antibody expression over almost 300 days. To investigate this phenomenon, we looked for the induction of antibodies against this human construct expressed in mice. We observed the development of anti-HER2dMAb antibody in sera after treatment with HER2dMAb (Supplemental Figure 1F), which may contribute to its decline over time.

Generation, expression, and cytotoxicity of HER2BiTE. BiTEs have 2 binding antibody fragments (single-chain variable fragments, scFvs) and are developed so that 1 of them engages the targeted tumor antigen while the other engages the immune system through binding to $\mathrm{CD} 3$, driving $\mathrm{T}$ cell activation at the tumor.

We reasoned that an optimized HER2dMAb that was engineered for in vivo expression would also be an excellent starting point for an engineered HER2DBiTE. Accordingly, we fused the scFv of the HER2dMAb with the scFv of an optimized sequence encoding anti-CD3 (modified UCHT1) (Figure 4A). This HER2BiTE was efficiently expressed in vivo upon injection and CELLECTRA (Inovio) electroporation into the mouse tibialis anterior muscle (Figure 4B). The new HER2DBiTE retained binding to HER2 and bound to CD3 (Supplemental Figure 2, A and B). Importantly, although 
A

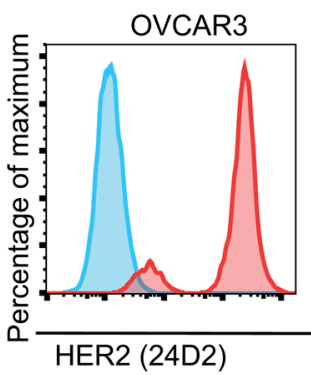

HER2 (24D2)
OVCAR3

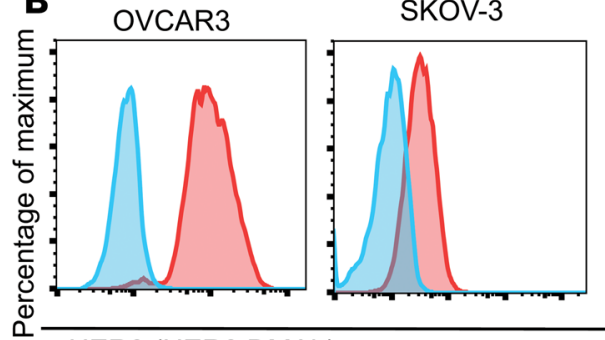

SKOV-3
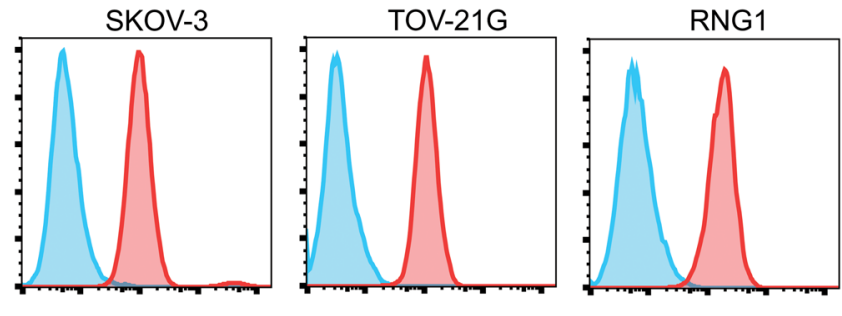

TOV-21G
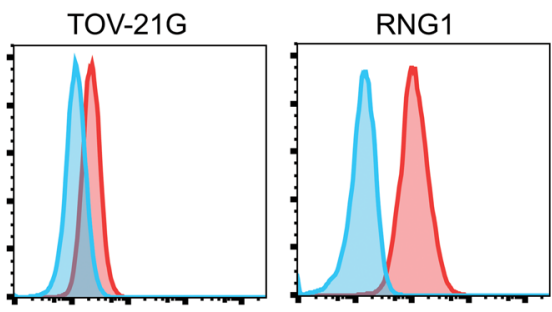

HER2 (HER2 DMAb)

C
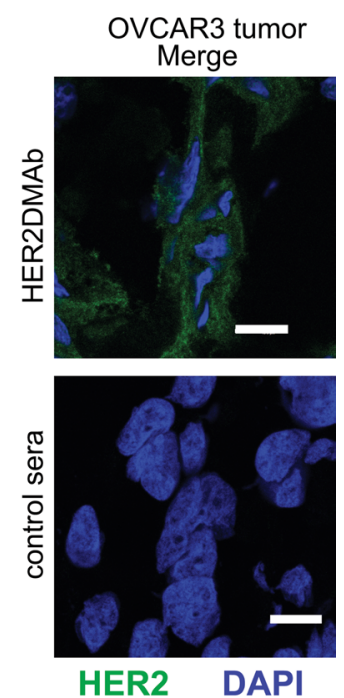

Figure 2. HER2dMAb binds to HER2 in ovarian cancer. HER2 expression in ovarian carcinoma cell lines OVCAR3, SKOV3, TOV-21G, and RNG1 by flow cytometry using (A) anti-HER2 antibody 24D2 and (B) sera from mice expressing HER2dMAb (representative of 2 independent experiments). (C) Immunofluorescence imaging of an OVCAR3 tumor stained with sera from HER2dMAb-expressing mice $(n=3$ mice). Scale bar: $10 \mu \mathrm{m}$.

the stimulation UCHT1 provides has been reported capable of killing T cells, we didn't observe an increased proportion of apoptosis or difference in T cell numbers when we cocultured OKT3 cells with HER2DBiTE as compared with just control in the presence of HER2 ${ }^{+}$cells (Supplemental Figure 2, C and D). To determine the functionality of the HER2DBiTE expressed in vivo, we cultured sera from mice injected with HER2DBiTE or empty vector with HER2+ ovarian cancer cells and T cells. Sera from mice after HER2DBiTE treatment showed T cell activation (Supplemental Figure 2, E-G) and an efficient dose-dependent cytotoxicity of OVCAR3 and CAOV3 cells. No cytotoxicity was found upon incubation with sera from empty vector-treated mice or was observed in the absence of $\mathrm{T}$ cells (Figure $4 \mathrm{C}$ and Supplemental Figure $2 \mathrm{H}$ ). Incubation of $\mathrm{T}$ cells with OVCAR3 at a 1:5 ratio with 5\% sera (5 $\mu 1$ in $100 \mu \mathrm{l})$ from treated mice showed that the DBiTEs exhibited potent activity for approximately 4 months (Figure 4D). As in HER2DBiTE, we observed generation of anti-HER2DBiTE antibodies, which could in part be responsible for the circulating levels over time (Supplemental Figure 2I). To determine the DBiTE antitumor activity in vivo, we challenged NOD/SCID- $\gamma$ (NSG) mice with OVCAR3. We treated mice with a single administration of $200 \mu \mathrm{g}$ of HER2DBiTE or empty vector a day after tumor implantation. Two weeks after tumor inoculation, when tumors were approximately $50 \mathrm{~mm}^{3}$, we injected 10,000,000 PBMCs intraperitoneally into each mouse. HER2DBiTE treatment significantly affected tumor progression (Figure 4E), with tumor regression or tumor elimination observed in 8 out of 10 tumors while no tumor impact was observed in the control group (Figure 4F). No in vivo effect of HER2DBiTE was observed in the absence of PBMCs (Supplemental Figure 2J).

\section{Discussion}

In this study we describe the generation of and therapeutic use of an optimized, synthetic DNA-encoded anti-HER2 $\mathrm{mAb}(\mathrm{dMAb})$ as well as a further DBiTE delivered in vivo as a cancer immunotherapy by adaptive electroporation.

dMAbs have a series of advantages over the traditional protein antibodies. First, DNA is stabler than proteins. This higher stability simplifies storage and shipping because of the current strict cold chain requirement for antibodies. These requirements can increase therapeutic costs and can limit in vivo product half-life (14). We show that intramuscular delivery of designed antibody-encoding DNA plasmids achieves stable plasma antibody concentrations for several months, limiting the requirement for multiple administrations, simplifying treatment strategies, as well as providing an improved expression profile. 
A
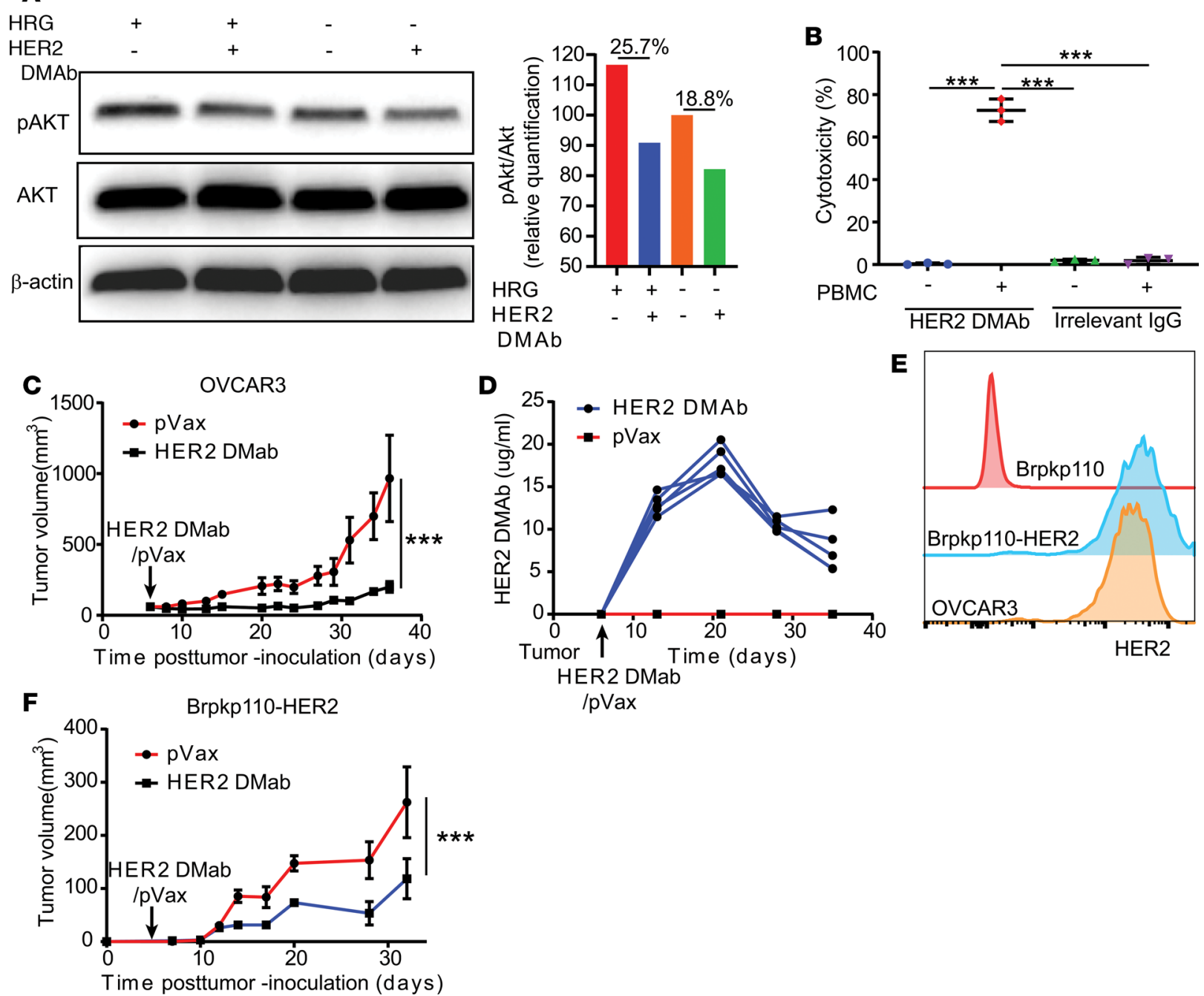

Figure 3. HER2dMAb blocks HER2 signaling, induces antibody-dependent cellular cytotoxicity, and delays cancer progression in vivo. (A) Western blot showing total Akt and phosphorylated Akt (p-Akt) and $\beta$-actin from OVCAR3 cells treated with or without HRG in the presence of HER2dMAb or control and quantification (representative of 3 independent experiments). (B) Histogram showing ADCC assay of HER2dMAb or irrelevant IgG with OVCAR3 (triplicates, representative of 3 independent experiments). (C) Growth curve of OVCAR3 tumors grafted into nude mice treated with HER2dMAb or empty vector ( 2 independent experiments, $n=5$ mice per group). (D) Levels of HER2dMAb in serum of OVCAR3-bearing mice treated with HER2dMAb or empty vector (representative of 2 independent experiments, $n=5$ mice per group). (E) Flow cytometry plot showing expression of HER2 by OVCAR3, Brpkp110, and Brpkp110-hHER2 tumor cells ( $n=3$ mice per group). (F) Growth curve of Brpkp110-hHER2 tumors grafted into C57BL/6 mice treated with HER2dMAb or empty pVax plasmid (representative of 2 independent experiments, $n=5$ mice per group). Two-way ANOVA, $t$ test, log-rank test. ${ }^{* *} P<0.001$.

Despite high antitumor activity, BiTE therapy has advanced slowly because these new tools have limitations due to manufacturing problems and half-life of approximately 2.1 hours. Its short half-life imposes BiTE therapy to be administered as continuous intravenous infusion for $4-8$ weeks per cycle (4). Recent experiments with an intravenously delivered RNA expressing BiTE show in vivo activity for up to 6 days (15). HER2DBiTE, delivered by a simple injection lasting just milliseconds, was expressed in vivo for approximately 4 months, resulting in sustained, dramatic antitumor activity. Synthetic DNA delivery of BiTEs could alleviate the burden generated by the short half-life of BiTE therapy and provide an important new application for this tool in cancer immunotherapy.

In this report we focus on targeting HER2 in ovarian cancer. A large study identified HER2 overexpression in $11.4 \%$ of ovarian tumors (11). Pertuzumab, unlike trastuzumab, does not require HER2 overexpression in the tumor cell for its antitumor activity (16), increasing the potential population sensitive to this therapy. Addition of pertuzumab to standard chemotherapy and trastuzumab in HER2 $2^{+}$breast cancer further improves progression and invasive disease-free survival in HER2 ${ }^{+}$breast cancer. In ovarian cancer, 


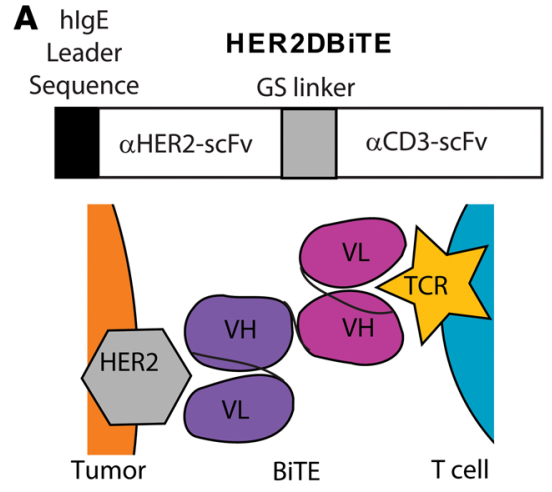

D

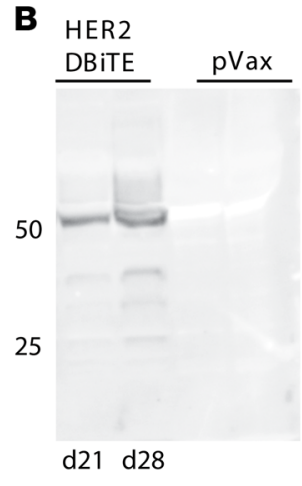

C

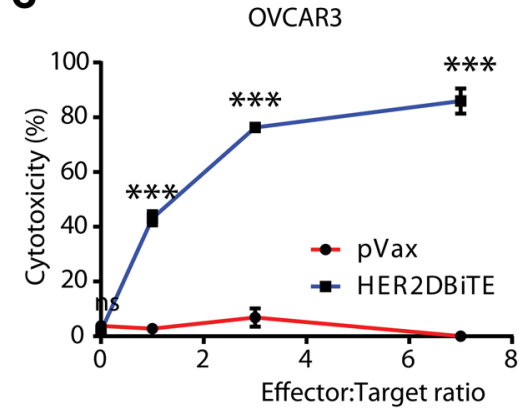

E

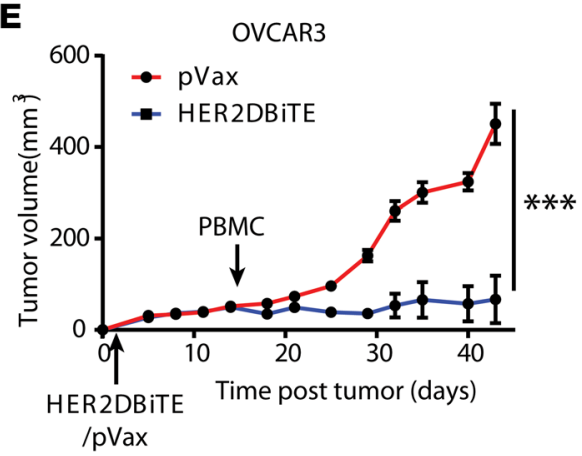

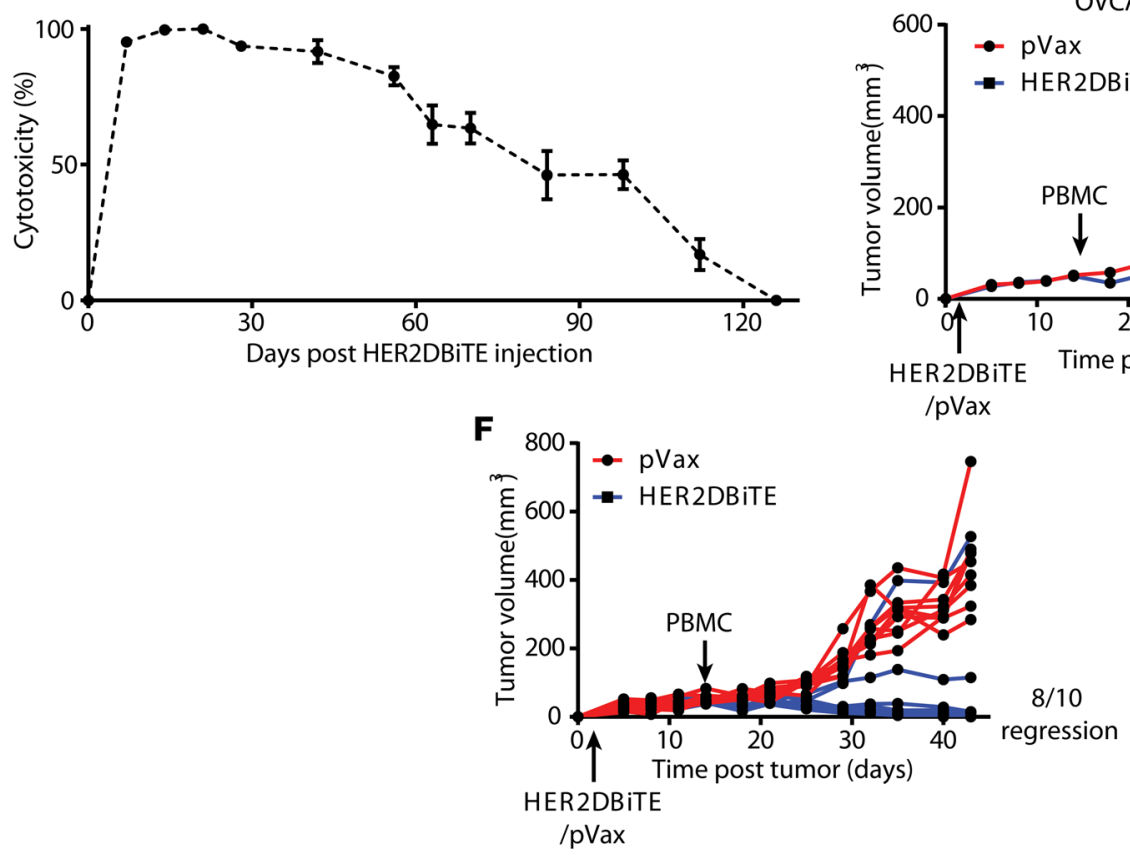

Figure 4. Generation, expression, and antitumor activity of HER2DBiTE. (A) Schematic of DNA construct encoding HER2DBiTE and cartoon of BiTE engaging HER2 and the TCR. GS, glycine-serine; VH, heavy chain variable region; VL, light chain variable region; TCR, T cell receptor. (B) Western blot of human IgG from $1 \mu \mathrm{l}$ of mouse sera electroporated with HER2DBiTE or pVax empty vector 21 and 28 days after DNA injection (triplicates, representative of 3 experiments). Numbers indicate molecular weight $(\mathrm{kDa})$. (C) In vitro cytotoxicity resulting from coculture of T cells with OVCAR3 cells at different ratios $(0,1,3$, and 7$)$ in the presence of sera from HER2DBiTE or pVax mice (2 independent experiments in triplicate). (D) In vitro cytotoxicity of sera from mice treated with HER2DBiTE after injection and electroporation of $100 \mu \mathrm{g}$ using OVCAR3 as the target (triplicates). (E) Average growth curve of OVCAR3 tumors grafted into NSG mice treated with HER2DBiTE or empty vector ( $n=10$ mice per group). (F) Individual growth curves of OVCAR3 tumors grafted into NSG mice treated with HER2DBiTE or empty vector ( $n=10$ mice per group). Two-way ANOVA. ${ }^{* *} P<0.001$.

pertuzumab has shown a trend toward increase in progression-free survival (17). The synthetic DNA platform production shown here would allow administration of pertuzumab as a dMAb as well as a DBiTE or their administration as a combination with DNA-encoded checkpoint inhibitors $(8,9)$. Such combination approaches have the advantage of a potentially higher antitumor effects using a simplified therapeutic regime that would favor adherence to treatment.

In this study we have also observed that despite long half-lives, antiantibody responses influence $\mathrm{dMAb}$ and DBiTE levels over time. Antibody humanization is able to reduce antiantibody responses in human therapies (18), and as we have reported, dMAb engineering can limit responses against anti-drug antibodies (ADAs) (19). We expect that implementation of this and other ways to prevent antiantibody responses will further contribute to positively influence dMAb circulating levels in future studies. Data from blinatumomab, the only BiTE approved to date, show that there is development of neutralizing ADAs in less than $1 \%$ of the patients treated (4). 
These studies demonstrate that HER2dMAbs can allow for several months of durable expression, which can drive antitumor activity. Further engineering of these expressed forms into BiTEs allows for a significant degree of potency in vivo. dMAb-engineered sequences can be a starting point for development of improved BiTEs when launched from DNA cassettes (HER2DBiTE) and can result in improved half-life extending their antitumor activity. These approaches appear valuable for additional exploration as tools for the treatment of ovarian as well as potentially other cancers.

\section{Methods}

Additional experimental details can be found in the supplemental material.

Animals and cell lines. C57BL/6 and Nu/J mice were purchased from The Jackson Laboratory. NSG mice were purchased from the Wistar Institute Animal Facility.

OVCAR3, CAOV3, SKOV3, and Brpkp110 cells were provided by J.R. Conejo-Garcia (Department of Immunology, Moffitt Cancer Center, Tampa, Florida). TOV-21G and RNG1 were provided by R. Zhang (The Wistar Institute). We generated OVCAR3 tumors by injecting 3,000,000 cells in the flank in Matrigel (Corning) diluted in PBS (50:50) as described previously (20). RD and 293T cells were purchased from ATCC. Human primary PBMCs, T cells, and monocytes were provided from healthy donors by the Human Immunology Core of the University of Pennsylvania.

Mice were treated by injecting 100-200 $\mu \mathrm{g}$ of DNA resuspended in 60-80 $\mu 1$ of water into the tibialis anterior muscle (30-40 $\mu \mathrm{l}$ per leg) with $200 \mathrm{IU} / \mathrm{ml}$ of hyaluronidase (MilliporeSigma), followed a minute after injection by electroporation with the CELLECTRA device (Inovio).

Design of HER2dMAb and HER2DBiTE. We engineered the HER2dMAb by encoding a codon-optimized sequence of the heavy and light chains of the anti-HER2 mAb pertuzumab. Both antibody chains were positioned in sequence separated by a P2A and furin cleavage site. We substituted their original leader sequence for the IgE leader sequence. We designed HER2DBiTE by encoding a codon-optimized scFv of HER2dMAb followed by the scFv of a modified UCHT1 anti-human CD3 antibody and adding an IgE leader sequence. Both constructs were subcloned into a modified pVAX expression vector (Figure 1A and Figure 4A), a nonreplicative, nonintegrating plasmid designed for efficient delivery of DNA vaccines and mAbs $(21,22)$.

We used empty modified pVAX plasmid as negative control.

Statistics. Differences between the means of experimental groups were calculated using a 2-tailed, unpaired Student's $t$ test or 1-way ANOVA where 2 categorical variables were measured. Repeated measures were analyzed using 2-way ANOVA. Error bars represent \pm SEM. Survival rates were compared using the log-rank test. All statistical analyses were done using GraphPad Prism 7.0. $P$ value less than 0.05 was considered statistically significant.

Study approval. Animal experiments were approved by the Institutional Animal Care and Use Committee at The Wistar Institute (protocol 112762).

\section{Author contributions}

APP, KM, and DBW conceived the study. APP and EKD designed the experiments. APP, EKD, MCW, ETR, XZ, PH, SHJ, KW, and XY performed the experiments. APP wrote the original manuscript, and APP, EKD, KM, and DBW reviewed and edited it. DBW and LJM acquired funding; DBW, LJM, and KM provided resources. DBW and KM provided supervision.

\section{Acknowledgments}

This work was supported by a University of Pennsylvania/Wistar Institute NIH Special Program of Research Excellence grant (P50 CA174523 to DBW), the Wistar National Cancer Institute Cancer Center (P30 CA010815), the W.W. Smith Family Trust (to DBW), the Basser Foundation (to DBW), and a grant from Inovio Pharmaceuticals (to DBW). EKD was supported by NIH award F32 CA213795. We would like to thank the Wistar Flow Cytometry Facility and Animal Facility for their technical assistance.

Address correspondence to: David B. Weiner or Kar Muthumani, The Wistar Institute, 3601 Spruce St., Philadelphia, Pennsylvania 19104, USA. Phone: 215.495.6882; Email: weiner@Wistar.org (D.B. Weiner); kmuthumani@Wistar.org (K. Muthumani). 
1. Coiffier B, et al. CHOP chemotherapy plus rituximab compared with $\mathrm{CHOP}$ alone in elderly patients with diffuse large-B-cell lymphoma. N Engl J Med. 2002;346(4):235-242.

2. Slamon DJ, et al. Use of chemotherapy plus a monoclonal antibody against HER2 for metastatic breast cancer that overexpresses HER2. N Engl J Med. 2001;344(11):783-792.

3. Viardot A, et al. Phase 2 study of the bispecific T-cell engager (BiTE) antibody blinatumomab in relapsed/refractory diffuse large B-cell lymphoma. Blood. 2016;127(11):1410-1416.

4. Zhu M, et al. Blinatumomab, a Bispecific T-cell Engager (BiTE(®)) for CD-19 targeted cancer immunotherapy: clinical pharmacology and its implications. Clin Pharmacokinet. 2016;55(10):1271-1288.

5. Muthumani K, et al. Rapid and long-term immunity elicited by DNA-encoded antibody prophylaxis and DNA vaccination against Chikungunya virus. J Infect Dis. 2016;214(3):369-378.

6. Flingai S, et al. Protection against dengue disease by synthetic nucleic acid antibody prophylaxis/immunotherapy. Sci Rep. 2015;5:12616.

7. Elliott STC, et al. DMAb inoculation of synthetic cross reactive antibodies protects against lethal influenza A and B infections. NPJ Vaccines. 2017;2:18.

8. Duperret EK, et al. Synthetic DNA-encoded monoclonal antibody delivery of anti-CTLA-4 antibodies induces tumor shrinkage in vivo. Cancer Res. 2018;78(22):6363-6370.

9. Perales-Puchalt A, Duperret EK, Muthumani K, Weiner DB. Simplifying checkpoint inhibitor delivery through in vivo generation of synthetic DNA-encoded monoclonal antibodies (DMAbs). Oncotarget. 2019;10(1):13-16.

10. Hollevoet K, De Smidt E, Geukens N, Declerck P. Prolonged in vivo expression and anti-tumor response of DNA-based antiHER2 antibodies. Oncotarget. 2018;9(17):13623-13636.

11. Bookman MA, Darcy KM, Clarke-Pearson D, Boothby RA, Horowitz IR. Evaluation of monoclonal humanized anti-HER2 antibody, trastuzumab, in patients with recurrent or refractory ovarian or primary peritoneal carcinoma with overexpression of HER2: a phase II trial of the Gynecologic Oncology Group. J Clin Oncol. 2003;21(2):283-290.

12. Franklin MC, Carey KD, Vajdos FF, Leahy DJ, de Vos AM, Sliwkowski MX. Insights into ErbB signaling from the structure of the ErbB2-pertuzumab complex. Cancer Cell. 2004;5(4):317-328.

13. Budzynski W, Radzikowski C. Cytotoxic cells in immunodeficient athymic mice. Immunopharmacol Immunotoxicol. 1994;16(3):319-346.

14. Hernandez I, et al. Pricing of monoclonal antibody therapies: higher if used for cancer? Am J Manag Care. 2018;24(2):109-112.

15. Stadler CR, et al. Elimination of large tumors in mice by mRNA-encoded bispecific antibodies. Nat Med. 2017;23(7):815-817.

16. Agus DB, et al. Targeting ligand-activated ErbB2 signaling inhibits breast and prostate tumor growth. Cancer Cell. 2002;2(2):127-137.

17. Kurzeder C, et al. Double-blind, placebo-controlled, randomized phase III trial evaluating pertuzumab combined with chemotherapy for low tumor human epidermal growth factor receptor 3 mRNA-expressing platinum-resistant ovarian cancer (PENELOPE). J Clin Oncol. 2016;34(21):2516-2525.

18. Harding FA, Stickler MM, Razo J, DuBridge RB. The immunogenicity of humanized and fully human antibodies: residual immunogenicity resides in the CDR regions. MAbs. 2010;2(3):256-265.

19. Khoshnejad M, et al. Development of novel DNA-encoded PCSK9 monoclonal antibodies as lipid-lowering therapeutics. Mol Ther. 2019;27(1):188-199.

20. Perales-Puchalt A, et al. Follicle-stimulating hormone receptor is expressed by most ovarian cancer subtypes and is a safe and effective immunotherapeutic target. Clin Cancer Res. 2017;23(2):441-453.

21. Sheets RL, et al. Biodistribution of DNA plasmid vaccines against HIV-1, Ebola, severe acute respiratory syndrome, or West Nile virus is similar, without integration, despite differing plasmid backbones or gene inserts. Toxicol Sci. 2006;91(2):610-619.

22. Ferraro B, Morrow MP, Hutnick NA, Shin TH, Lucke CE, Weiner DB. Clinical applications of DNA vaccines: current progress. Clin Infect Dis. 2011;53(3):296-302. 